\title{
Surgical management of hepato-pancreato-biliary cancers in Covid-19 pandemic: perspective from the developing world
}

\author{
Saleema Begum', Muhammad Rizwan Khan ${ }^{2}$ \\ ${ }^{1}$ Shaukat Khanum Memorial Cancer Hospital and Research Center, Pakistan \\ ${ }^{2}$ Aga Khan University Hospital, Pakistan
}

Keywords: COVID-19; HPB malignancies; Pakistan

\begin{abstract}
Coronavirus disease has dismantled the health care systems in the world and the situation is worse in the developing countries. One of the biggest challenges faced by clinicians in this situation is the optimal management of cancer patients. Hepato-pancreato-biliary [HPB] malignancies are the most aggressive cancers among other gastrointestinal cancers. Timely surgical intervention is the only cure for resectable tumours. However, resource limitations in hospitals, economic crisis and the lockdown in the current pandemic have forced clinicians to choose alternate non-surgical therapies to get temporary control of disease with the hope to provide definitive surgical management once the situation gets better. In this communication, we provide a summary of the management guidelines for HPB cancers and their application in the developing world.
\end{abstract}

\section{Background}

The spread of coronavirus disease [COVID-19] is rapid without respecting any boundaries. The death toll has reached up to 738,000 worldwide and seems to be rising daily [1]. The numbers of infected and critically sick patients are also expected to rise, threatening the lives of many individuals. This pandemic has already dismantled the best health care systems in the world, and they are struggling to cope with the rising trends in the coming days. The current health situation has forced clinicians to make difficult triage decisions to provide optimal health care [2]. One of the biggest challenges faced by clinicians is the optimal management of cancer patients. Unlike other health conditions, cancer care is complex and costly; requiring a multidisciplinary approach. Elective cancer surgeries have been suspended in most of the countries in the developed world due to severe strain on the resources, including ventilators. There are reports that the operating theatres have been converted into intensive care units to accommodate the influx of sick patients infected with COVID-19 [3]. This could result in the progression of cancers

Correspondence: Saleema Begum

E-mail: saleema 85@hotmail.com

(iD) https://orcid.org/0000-0002-3780-5465

Received: 14-05-2020 Accepted: 26-07-2020

DOI: http://doi.org/10.4038/sljs.v38i2.8699 with subsequent metastasis or obstruction in case of gastrointestinal cancers with an immediate threat to survival.

Hepato-pancreato-biliary [HPB] malignancies are the most aggressive cancers among other gastrointestinal cancers [4, 5]. Timely surgical intervention is the only cure for resectable tumours. However, this pandemic has challenged the provision of cancer care due to competing risks of death from cancer versus death from complications of a deadly virus. Patients with curable cancers may contract COVID-19 during treatment which might offset the advantage of cancer treatment. The study from China reported that cancer patients affected by COVID-19 were more likely to require ventilatory support, intensive care admission and the mortality rate was five times higher than their non-cancer counterparts. [6].

Also, inadequate supplies of personal protective equipment for surgeons and other healthcare providers in the operating room as well as the limited hospital capacity including ICU have contributed to the suspension of elective cancer procedures in both the developed and the developing world. In this communication, we have summarized the guidelines published for HPB cancers in this pandemic situation and their application in the local context of the developing world during this time of crisis.

\section{Recommendations for the treatment of Hepato-pancreato- biliary malignancies:}

HPB malignancies have been notorious for high morbidity and mortality with prolonged hospitalization and readmissions following surgery. The adverse effects are secondary to the technical complexity of operations, cachexia, and malnutrition due to underlying malignancy and age-related comorbidities which may be associated with higher perioperative morbidity in COVID-19 positive asymptomatic patients.

Additionally, these operations have the potential to consume a significant amount of hospital resources. Considering their aggressiveness, managing them according to routine cancer guidelines may pose a huge burden on resources of the hospital in the times of COVID-19 pandemic. Oncology societies around the world have been efficient enough to alter cancer management during COVID-19 pandemic and have 
published guidelines for the management of various cancers. This has helped the healthcare providers across the globe to practice oncology efficiently and with uniformity.

Society of surgical oncology [SSO] has referred HPB malignancies as biologically aggressive tumours and not just 'elective' operations. The decision on operating needs to be done according to the input from the multidisciplinary care providers, mode of presentation and biology of the disease, ICU/ventilator bed availability and utilization and the projected number of COVID-19 patients influx. During the acute phase I, when hospital resources including ICU and ventilator are not exhausted, all patients with upfront resectable pancreatic and biliary tumours, and patients who have completed neoadjuvant therapy including those with colorectal liver metastasis should be operated. Neoadjuvant therapy should be considered for newly diagnosed pancreatic adenocarcinoma and large intrahepatic cholangiocarcinoma requiring major liver resection. Similarly, ablation, regional therapy or neoadjuvant therapy should be the initial approach for hepatocellular carcinoma [HCC]. During phase II, when intensive care and operating room resources are restricted, only tumours with gastric outlet obstruction or bleeding which are not amenable to endoscopic intervention, symptomatic hormone-producing tumours like insulinomas and surgical complications not feasible to interventional approach should be operated. All other pancreaticobiliary cases should be offered neoadjuvant therapy and liver tumours should be treated with ablation and locoregional therapies. During phase III, when hospital resources are all directed to COVID-19 patients and intensive care beds and operating room resources are consumed bleeding tumours that cannot be managed with interventional therapy, acute tumour perforation and surgical complications not feasible to interventional approach should be operated [7].

American College of Surgeons has published detailed guidelines for elective cancer surgery during the acute and recovery phase of COVID-19 pandemic. During the phase I, pancreatic and ampullary surgeries should be performed in patients with limited comorbidities with expected short length of stay and utilization of fewer hospital resources. Patients with potentially curable pancreatic cancers with or without neoadjuvant therapy and patients with biliary and gastric obstruction should be operated. All other cases should be offered neoadjuvant therapy and interventional procedures. During phase II, all but emergent lifesaving HPB operations should be deferred due to limited operating room supplies, ICU, and ventilators. Only emergencies associated with these cancers should be palliated with nonsurgical methods. The endoscopic or percutaneous route should be used for biliary drainage in biliary sepsis, endoscopic enteral stenting for gastric outlet obstruction, and radiologic management for bleeding tumours. All patients with potentially curable pancreatic, duodenal and ampullary cancers should be offered neoadjuvant treatment [8].

European Society of Medical Oncology has recommended surgery for patients with primary or post neoadjuvant resectable pancreatic cancers, and borderline cancers are not fit for neoadjuvant on high priority. For patients with bile duct obstruction in non-resectable or metastatic cancers, cholangitis and bilirubin levels more than $250 \mu \mathrm{mol}$, or nonresectable localized cancers allocated to neoadjuvant or palliative treatment should be decompressed endoscopically with biliary stents. High priority should be given to patients with postoperative complications like an anastomotic leak, bleeding and fistulae to offer surgical or image-guided procedures. For HCC, the liver transplant should only be offered to patients with a poor short-term prognosis, a high model for end-stage liver disease [MELD] score and HCC at the upper limit of Milan criteria on high priority. Similarly, surgical intervention with curative intent has been advised for patients with large curatively resectable HCC lesions [9].

Despite these consensus guidelines published by the experts given the current situation, management of HPB malignancies is not straight forward. From a clinical standpoint, aggressive tumour biology, the complexity of procedures with associated high morbidity and mortality, and added risk of becoming affected by a coronavirus and its complications challenge the caregivers to undertake these procedures. There are suggestions that opting non-surgical management like initiating or continuing neoadjuvant chemotherapy for curable cancers can increase the risk of COVID-19 and other infections due to immune suppression [3] with the added risk of disease progression. Another consideration in the management of such patients is the availability of local hospital resources for the evolving situation [10]. Cancer societies have modified their management guidelines regarding the available hospital resources in different phases of the pandemic.

\section{Perspective from Pakistan}

Where COVID-19 has shaken the best health care systems in the world, the situation in developing countries is equally alarming. Unlike other developing countries, where a significant portion of gross domestic product [GDP] is allocated to maintain and improve healthcare, Pakistan only spends two percent of its GDP on health. Per statistics, one doctor is available to treat 1073 people, only one bed available per 1600 people, the number of registered nurses is only 90,000, and approximately 4000 ventilators are available for a population of 220 million [11]. The total number of confirmed COVID-19 positive cases are more than 284,000 till date [12] but the actual number of cases including 
asymptomatic patients is still unknown due to lack of testing capacity, and only limited patients are being tested daily [13]. According to experts, the number is expected to peak in the next few months, which is going to increase pressure on an already overburdened health care system.

With relevance to HPB malignancies, only a few centres in the country offer integrated services to the entire population of 220 million, located in three major cities of Pakistan. Given current COVID-19 pandemic, the country has been in a state of partial lockdown for more than four weeks now and hospitals are already overburdened with the influx of COVID positive patients requiring intensive care. This has resulted in a temporary suspension of elective HPB surgeries to provide intensive care beds and ventilators to complicated COVID-19 positive patients [13]. Providing neoadjuvant chemotherapy for operable cancers for disease control to operate them later in such situations is not an easy task either. Lockdown has deprived poor patients of using public transport to reach hospitals, the economic crisis has affected poor patients who can no longer afford expensive chemotherapy and those who can get chemotherapy treatment are at risk of contracting COVID-19 due to immunosuppression.

With the increasing number of COVID-19 cases day by day, the stage of operable HPB malignancies are expected to progress or metastasize due to definitive treatment delays.

In the current situation, the management guidelines for HPB cancers have been modified and the priority is to save lives. Health care system around the world is forced to prioritize treatment and take difficult decisions. Management plans need to be tailored according to available resources by HPB units to deliver the best management to patients in the time of crisis. As the duration of the pandemic is unpredictable and the country is still in phase I of the pandemic, few HPB units in the country should be dedicated to providing definitive surgical treatment to selected patients with limited comorbidities and shorter expected length of hospital stay.

This is particularly applicable to young patients whose survival may be seriously compromised due to delays in the provision of definitive care. It is strongly suggested that the developing countries should focus on developing local guidelines considering the local health facilities and the impact of the pandemic on the health care system. There is a need to have a balanced approach in offering the surgical and non-surgical therapies to HPB cancer patients with involvement of multidisciplinary teams. Due to limited resources compromised by the corona pandemic, only a well thought out approach can help save precious lives in this difficult situation.
All authors disclose no conflict of interest. The study was conducted in accordance with the ethical standards of the relevant institutional or national ethics committee and the Helsinki Declaration of 1975, as revised in 2000 .

\section{References}

1. Coronavirus Update [Live]. [Accessed August 11, 2020]. Available at: https:/www.worldometers.info/coronavirus/

2. Schrag D, Hershman DL, Basch E. Oncology Practice During the COVID-19 Pandemic. JAMA. 2020 Apr 13. https://doi.org/10.1001/jama.2020.6236

3. Burki TK. Cancer guidelines during the COVID-19 pandemic. The Lancet Oncol. 2020 Apr 2. https://doi.org/10.1016/S1470-2045(20)30217-5

4. Waisberg DR, Pinheiro RS, Nacif LS, et al. Resection for intrahepatic cholangiocellular cancer: new advances. Transl Gastroenterol Hepatol. 2018;3.

https://doi.org/10.21037/tgh.2018.08.03

5. Weledji EP, Enoworock G, Mokake M, Sinju M. How grim is pancreatic cancer? Oncol Rev. 2016 Apr 15;10[1]. https://doi.org/10.4081/oncol.2016.294

6. Liang W, Guan W, Chen R, et al. Cancer patients in SARS-CoV-2 infection: a nationwide analysis in China. Lancet Oncol 2020; 21:335-7. https://doi.org/10.1016/S1470-2045(20)30096-6

7. Resource for management options of GI and HPB cancers during COVID-19.[Accessed May 01, 2020]. Available at: https://www.surgonc.org/wp-content/uploads/2020/04/GI-andHPB-Resource-during-COVID-19-4.6.20.pdf

8. COVID-19 Guidelines for Triage of Cancer Surgery Patients. [ Accessed May 01, 2020]. Available at:

https://www.facs.org/covid-19/clinical-guidance/electivecase/cancer-surgery.

9. Cancer patient management during the COVID-19 pandemic. [Accessed May 03, 2020]. Available at

https://www.esmo.org/guidelines/cancer-patient-managementdu ring-the-covid-19-pandemic.

10SAGES-AHBPA Recommendations regarding surgical management of HPB cancers during the response to COVID-19 pandemic crisis. [Accessed May 03, 2020]. Available at: https://www.sages.org/author/sages-webmaster/

11.Story of neglect: Another grim year for healthcare in Pakistan. The express tribune. [Accessed May 02,2020].

Available at:

https://tribune.com.pk/story/2129467/1-story-neglect-anothergrim-year-healthcare-pakistan/?amp=1

12.See the real-time Pakistan and worldwide Covid-19 situation! [Accessed August 11, 2020]. Available at: http://covid.gov.pk/

13. Govt decides to enhance Covid-19 testing capacity. Accessed April, 24,2020]. Available at:

https://www.dawn.com/news/1547323/govt-decides-to-enhancecovid-19-testing-capacity

14.Yusuf A. Cancer care in the time of COVID-19- a perspective from Pakistan. Ecancer2020,14:1026 https://doi.org/10.3332/ecancer.2020.1026 